\title{
Hyperspectral Sensing Techniques Applied to Bio-masses Characterization: The Olive Husk Case
}

\author{
Giuseppe Bonifazi and Silvia Serranti \\ Dipartimento di Ingegneria Chimica Materiali Ambiente \\ Sapienza - Università di Roma \\ giuseppe.bonifazi@uniromal.it, silvia.serranti@uniromal.it
}

\begin{abstract}
Olive husk $(\mathrm{OH})$ quality, in respect of constituting particles characteristics (olive stones and pulp residues as result after pressing), represents an important issue. $\mathrm{OH}$ particles size class distribution and composition play, in fact, an important role for $\mathrm{OH}$ utilization as: organic amendment, bio-mass, food ingredient, plastic filler, abrasive, raw material in the cosmetic sector, dietary animal supplementation, etc. . OH is characterised by a strong variability according to olive characteristics and olive oil production process. Actually it does not exist any strategy able to quantify $\mathrm{OH}$ chemicalphysical attributes versus its correct utilisation adopting simple, efficient and low costs analytical tools. Furthermore the possibility to perform its continuous monitoring, without any samples collection and analysis at laboratory scale, could strongly enhance $\mathrm{OH}$ utilization, with a great economic and environmental benefits. In this paper an analytical approach, based on HyperSpectral Imaging (HSI) is presented. HSI allows to perform, also on-line, a full quantification of $\mathrm{OH}$ characteristics in order to qualify this product for its further re-use, with particular reference as bio-mass. HSI was applied to different samples of $\mathrm{OH}$, characterized by different moisture, different residual pulp content and different size class distributions. Results are presented and critically evaluated.
\end{abstract}

\section{Introduction}

Olives can be considered as the most cultivated fruit crop in the world. The Mediterranean region represent one of the most important and the olive production represents not only a source of income but also it plays an important role at environmental and heritage level [1]. The main producers are located in the Mediterranean and Middle East regions providing $98 \%$ of the total cultivated surface area and $99 \%$ of the total olive fruit production [2]. The world production of olives, for the year 2007, was 17.4 Mt, of which 12.6 Mt came from Europe [3]. Olive oil organoleptic properties, as well as its healthy-diet characteristics, strongly contributed to spread out the consumption all over the world and not only in the Mediterranean areas where it has been consumed for innumerable generations. Olive oil world output in 2007 approached $3 \mathrm{Mt}$, with the European Union (EU) being the leading producer and consumer, thanks to the substantial contribution of Spain, Italy, Greece and Portugal [3]. 
Olives are not only utilized to produce oil, but they can be directly consumed as "table olives". Table olives annual world production is about $2 \mathrm{Mt}$, one-third of which comes from the EU, followed by Egypt (20\%), Turkey (12\%) and a long list of progressively minor producers [4]. The manufacture of olive oil yields, in addition to the actual oil $(20 \%)$, a semi-solid cake $(30 \%)$ and an aqueous liquor $(50 \%)$. The crude olive cake, composed of olive pulp and olive stone. This latter product is usually dried, at $60^{\circ} \mathrm{C}$ by rotary drums, and then processed, utilizing an extraction process by hexane to recover the residual oil. At the end of the process an olive stone rich material with low moisture content is obtained: the olive husk $(\mathrm{OH})$ [2].

The process currently utilized to produce olive oil are mainly three, that is: i) the discontinuous traditional pressing system, ii) the three-phase continuous process and iii) the two-phase continuous process.

The discontinuous process represents the oldest method. A pressure is applied to stacked filter mats, smeared with paste, that alternate with metal disks; a central spike allows the expressed oil and water (olive juice) to exit. The machinery, however, is cumbersome, the process requires more labour than other extraction methods, the cycle is not continuous, and the filter mats can easily become contaminated.

In the three-phase continuous process large horizontal centrifuges (decanters) are utilized. The decanters spin at approximately $3.000 \mathrm{rpm}$. Centrifugal force moves the heavier solid materials to the outside. A lighter water layer is formed in the middle with the lightest oil layer on the inside. Decanters separate the paste into a relatively dry solid, fruit-water, and oil. Water is added to this system to get it to flow through the decanter. A minimum quantity of water is added to separate the solid material better and to retain water-soluble polyphenols as much as possible.

The two-phase continuous process works following the same principle as 3-phase decanters except that the solid and fruit-water exit together. No water needs to be added to the 2-phase system.

The discontinuous process is being progressively replaced, first by the continuous three-phase system and then by the two-phase system. The two-phase system practically almost doubles the amount of "solid" residue that further presents a limited storage life and high transportation costs. As a consequence, it is not a profitable feedstock for seed-oil extraction, posing a problem of waste disposal [5]. In order to overcome this constraint, some two-phase olive oil industries have implemented a stage for recovering the $\mathrm{OH}$, which is subsequently used as a fuel in bakeries, ceramic plants, as well as in the olive oil and seed-oil mills themselves [6]. Because of its high heating power (heat of combustion of about $4.1 \mathrm{kcal} / \mathrm{kg}$ ), $\mathrm{OH}$ finds application mostly in thermal processes being used for power generation in the electricity sector and for space calefaction in residential and commercial buildings [7]. Alternatively, it can be thermally degraded by pyrolysis and gasification to produce syngas, used as a fuel to produce electricity or steam or as a basic chemical feedstock in the petrochemical and refining industries [8] [9] [10] [11].

In this paper the possibility offered by a new technology, based on HyperSpectral Imaging (HSI) based sensors, and related detection architectures, to perform an evaluation of $\mathrm{OH}$ quality in respect of constituting particles characteristics (olive stones and pulp residues as result after processing). $\mathrm{OH}$ particles size class distribution and composition play, in fact, an important role for the use of such a kind of product as biomass: both for direct burning and/or for its utilization in digesters for 
the production of gas. The need to develop simple analytical tools able to perform a full quantification of olive husks characteristics addressed to their use as biomasses, not requiring a physical collections of the samples, and their further analysis at laboratory scale, it is quite important to set up handling strategies for a fast and reliable $\mathrm{OH}$ quality assessment. The possibility to develop such new marketacceptable characterization procedures could strongly enhance the optimal utilization of this waste product with great environmental advantages.

\section{The HyperSpectral Imaging (HSI)}

HyperSpectral Imaging (HSI), known also as chemical or spectroscopic imaging, is an emerging technique that combines the imaging properties of a digital camera with the spectroscopic properties of a spectrometer able to detect the spectral attributes of each pixel in an image. Thus, a hyperspectral image, is a three dimensional dataset with two spatial dimensions and one spectral dimension.

HSI was originally developed for remote sensing applications [12] but has found application in such diverse fields as astronomy [13] [15], agriculture [15] [16] [17], pharmaceuticals [18] [19] [20] and medicine [21] [22] [23]. Some advantages of HSI over conventional true color imaging (RGB), near infrared imaging (NIR) and multispectral imaging (MSI) are outlined in Table 1. HSI based architectures, in addition to spatial information, can provide spectral information in a wide wavelength range for each pixel of the image. Hyperspectral images are, in fact, made up of hundreds of contiguous wavebands for each spatial position of a target studied.

Each pixel in a hyperspectral image contains the spectrum of that specific position. The resulting spectrum acts like a fingerprint which can be used to characterise the composition of that particular pixel. Hyperspectral images, known as hypercubes [24], are three-dimensional blocks of data, comprising two spatial and one wavelength dimension (Figure 1). The hypercube allows for the visualization of specific attributes (i.e. physical, chemical, biological, etc.) characteristics of a specific sample, separated into particular areas of the image, since regions of a sample with similar spectral properties have similar "chemical" composition.

It is currently unfeasible to obtain information in all three-dimensions of a hypercube simultaneously; one is limited to obtaining two dimensions at a time, then creating a three-dimensional image by stacking the two dimensional "slices" in sequence. There are two conventional ways to construct a hypercube, the first one known as the "staring imager" configuration and the second known as "pushbroom" acquisition.

The "staring imager" configuration involves keeping the image field of view fixed, and obtaining images one wavelength after another. Hypercubes obtained using this configuration thus consist of a three-dimensional stack of images (one image for each wavelength examined), stored in what is known as the Band Sequential (BSQ) format. Wavelength in the "staring imager" configuration is typically moderated using a tuneable filter, Acousto-optic Tuneable Filters (AOTFs) and Liquid Crystal Tuneable Filters (LCTFs) are the two most predominantly employed. AOTFs have been used in the construction of commercially available NIR-CI systems [15]. The main advantages of AOTFs are good transmission efficiency, fast scan times and large spectral range. On the other hand, LCTFs show greater promise for filtering of 
Raman images, due to superior spectral bandpass and image quality [16]. "Staring imager" instruments incorporating tuneable filters have found a number of applications in pharmaceutical quality control [9] [17], their lack of moving parts represents an advantage in many situations.

Table 1. Comparison of RGB imaging, NIR spectroscopy (NIRS), multispectral imaging (MSI) and hyperspectral imaging (HSI) [13]

\begin{tabular}{lcccc}
\hline Feature & RGB imaging & NIRS & MSI & HSI \\
\hline Spatial information & yes & no & yes & yes \\
\hline Spectral information & no & yes & low & yes \\
\hline Multi-constituent information & low & yes & low & yes \\
\hline Sensitivity to minor components & no & no & low & $y \boldsymbol{y} s$ \\
\hline
\end{tabular}

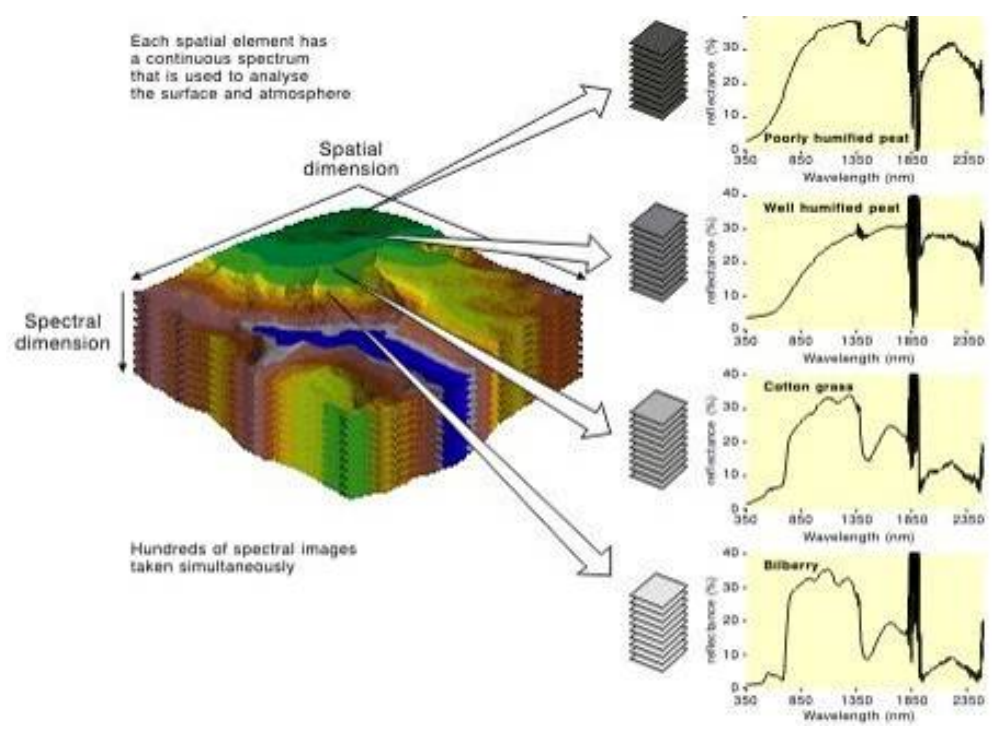

Fig. 1. Example of four land covers identified adopting hyperspectral measurement [http://www. sed.manchester.ac.uk/.../uperu/project4.htm]. On the left a hypercube of data with spatial location given by the $\mathrm{x}$ and $\mathrm{y}$ axes and spectral information contained along the z-axis is reported. The spectral distribution of the different components that make up that spectrum (along with their physical origin) are shown on the right hand side.

The "pushbroom" [18] configuration is based on the acquisition of simultaneous spectral measurements from a series of adjacent spatial positions, this requires relative movement between the object and the detector. Some devices produce hyperspectral images based on a point step and acquire mode: spectra are obtained at single points on a sample, then the sample is moved and another spectrum taken. Hypercubes obtained using this configuration are stored in what is known as the $\mathbf{B}$ and $\mathbf{I}$ nterleaved by Pixel (BIP) format. Advances in detector technology have reduced the time 
required to acquire hypercubes. Line mapping instruments record the spectrum of each pixel in a line of sample which is simultaneously recorded by an array detector; the resultant hypercube is stored in the $\mathbf{B}$ and $\mathbf{I}$ nterleaved by Line (BIL) format. This method is particularly well suited to conveyor belt systems, and may therefore be more practicable than the former for industry applications. The HSI detection architecture that has been utilized in the experimental tests reported in the following, belongs to this class of instruments.

Table 2. Technical characteristics of the ImSpector ${ }^{\mathrm{TM}}$ V10E

\begin{tabular}{|c|c|}
\hline Sensor & $\begin{array}{l}\text { - 2/3" CCD Array } 780 \times 580, \\
\text { - firewire digital output, } \\
\text { - pixel resolution: } 12 \text { bit. }\end{array}$ \\
\hline Spectral range & $400-1000 \mathrm{~nm}$ \\
\hline Spectral resolution & $2.8 \mathrm{~nm}$ \\
\hline Smile & $<1.5 \mu \mathrm{m}$ \\
\hline Keoneyst & $<1 \mu \mathrm{m}$ \\
\hline Entrance slit & $30 \mu \mathrm{m} \times 14.2 \mu \mathrm{m}$ \\
\hline Image size & $6.5 \mathrm{~mm} \times 14.2 \mathrm{~mm}$ \\
\hline Numerical aperture & $\mathrm{F} / 2.4$ \\
\hline Illuminant & $\begin{array}{l}\text { - anodised Aluminum cylinder, } \\
\text { - Barium sulfate internal coating, } \\
\text { - d/O illumination and viewing conditions, } \\
\text { - adjustable height and distance, } \\
\text { - } 150 \mathrm{~W} \text { cooled halogen lamp, } \\
\text { - stabilised power source. }\end{array}$ \\
\hline
\end{tabular}

\subsection{HSI Architecture, Equipment and Set Up}

Pushbroom HIS bases architecture is typically constituted by the following units: optics, spectrograph, camera, acquisition system, translation stage, energizing source (illumination) and computer (Figure 2). The characteristics of the camera, the spectrograph and the illumination conditions determine the spectral range of the detection architecture. A VIS-NIR system, on of the most utilized, typically ranges between 400 and $1000 \mathrm{~nm}$, and utilize Charge Coupled Device (CCD) cameras or Complementary Metal Oxide Semiconductor (CMOS) sensors. Longer wavelength systems require more expensive IR focal-plane array detectors with appropriate spectrograph which operates in the IR region.

The sample/target is usually diffusely illuminated by a tungsten-halogen or LED source. A line of light reflected from the sample enters the objective lens and is separated into its component wavelengths by diffraction optics contained in the spectrograph. A two dimensional image (spatial dimension-wavelength dimension) is then formed on the camera and saved on the computer. The sample is moved past the objective lens on a motorized stage and the process repeated. Two dimensional line images acquired at adjacent points on the object are stacked to form a threedimensional hypercube which may be stored on a PC for further analysis. 
The acquisition device utilised in this study is a ImSpector ${ }^{\mathrm{TM}} \mathrm{V} 10 \mathrm{E}$ operating in the spectral range of 400-1000 $\mathrm{nm}$ with a spectral resolution of $2.8 \mathrm{~nm}$. The details of the acquisition architecture are reported in Table 2 . The device is fully controlled by a PC unit equipped with the Spectral Scanner ${ }^{\mathrm{TM}}$ v.2.3 (SSOM, 2008) acquisition/preprocessing software.

The spectrograph is constituted by optics based on volume type holographic transmission grating [20]. The grating is used in patented Prism-Grating-Prism construction (PGP element) characterized by high diffraction efficiency, good spectral linearity and it is nearly free of geometrical aberrations due to the on-axis operation principle. A collimated light beam is dispersed at the PGP so that the central wavelength passes symmetrically through the grating and prisms and the short and longer wavelengths are dispersed up and down compared to central wavelength (Figure 3).

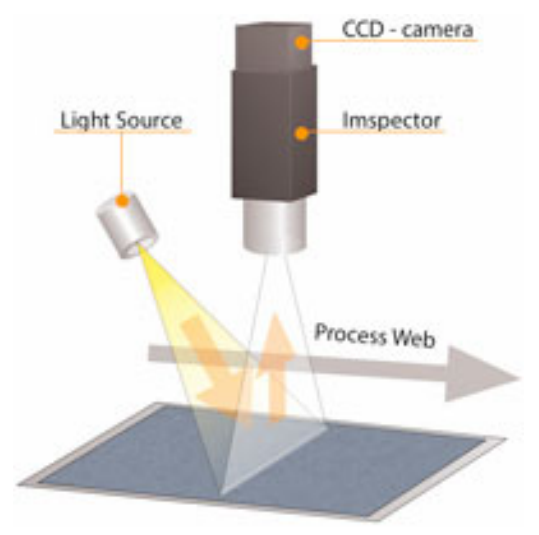

Fig. 2. Architecture set-up utilized to perform a progressive and continuous surface spectra acquisition

A digital image is thus acquired: each column represents the discrete spectrum values of the corresponding element of the sensitive linear array. Such an architecture allows, with a "simple" arrangement of the detection device ("scan line" perpendicular to the moving direction of the objects) to realize a full and continuous control (Figure 2).

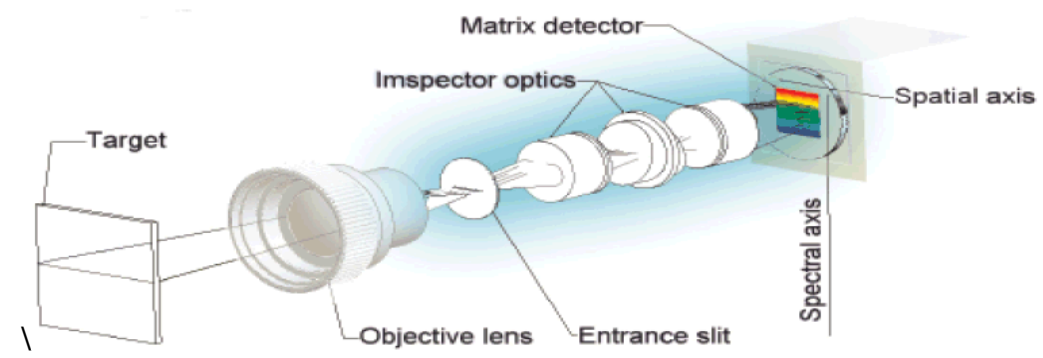

Fig. 3. Operating principle of ImSpector ${ }^{\mathrm{TM}}$ 
A line lighting, as energizing source with uniform spatial distribution, was used. Calibration was performed in three steps: i) spectral axis calibration with spectral lamps; ii) dark image acquisition and iii) measurements of "white reference image". After the calibration phase: i) the image spectra is acquired and ii) the reflectance $\left(\mathrm{R}_{\mathrm{ci}}\right)$ (at wavelengths $i$ and spatial pixels $c$ of interest) is computed:

$$
\mathrm{R}_{\mathrm{ci}}=\left[(\text { sample })_{c i}-(\text { dark })_{c i}\right] /\left[(\text { white })_{c i}-(\text { dark })_{c i}\right]
$$

such a procedure enables to compensate the offset due to CCD dark current and separates the sample reflectance from the system response.

\section{Experimental}

\subsection{Sample Set Selection}

Different $\mathrm{OH}$ samples, coming from the same oil-waste mill products have been collected. Each sample was characterized according to $\mathrm{OH}$ utilization as bio-mass. Moisture content, as well as size class distribution, were thus determined for each sample. Playing both the attributes a preeminent role in the combustion process. HSI based investigations have been then carried out, on the same samples, to verify the possibility to utilize this technique to derive $\mathrm{OH}$ moisture and size class distribution characteristics, directly looking at $\mathrm{OH}$ flow streams as resulting from an oil mill and/or fed to a thermal process. Furthermore the hyperspectral approach was applied to perform a quality control of $\mathrm{OH}$ according to its composition, that is relative presence of olive stones, pulp, olive peel and leaves inside the $\mathrm{OH}$. All the efforts have been thus addressed to develop and set up strategies able to reduce analytical costs improving the speed of the analytical controls and/or to simplify the procedures in terms of possible on-line implementation of fast and robust classification procedures oriented to develop innovative quality control strategies as well as innovative $\mathrm{OH}$ certification criteria.

\subsection{Spectra Acquisition, Detection and Analytical Logics Implementation}

Spectra related to the different investigated samples were collected adopting the acquisition architecture previously described (Figure 2). Such a strategy was adopted because it mimics, at laboratory scale, the real behaviour of the control architecture at industrial scale, that is, the progressive and continuous horizontal translation of the sample and the "synchronized" acquisition of the spectra at a pre-established step, allowing this way a tuning of the detection/inspection frequency of the $\mathrm{OH}$. Analyses have been performed to verify the fulfilments of different goals, that is:

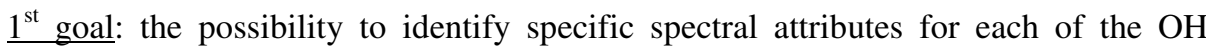
according to their intrinsic chemical-physical characteristics. Starting from these information, analysis have been carried out performing a characterization of the "shape" of the entire detected spectra and/or identifying peaks or valley characterising the spectral firm. 
$2^{\text {nd }}$ goal: the definition of fast, reliable and robust recognition-classification procedures, based on different logics as: i) specific wavelengths intensity ratio analysis, ii) multiple single band intensity comparison at specific wavelengths and iii) spectral firms correlation, in order to built provisional models able to predict $\mathrm{OH}$ moisture content and/or $\mathrm{OH}$ size class attributes starting from the collected spectra, allowing this way the possibility to certificate $\mathrm{OH}$ in respect of the two previously mentioned characteristics. To reach this latter goal a Partial Least Squares (PLS) regression approach was followed [25] [26] [27] [28] [29]. PLS is a technique that generalizes and combines features from Principal Component Analysis (PCA) and $\mathbf{M} u \mathbf{L}$ tiple Regression (MLR). It is particularly useful when it needs to predict a set of dependent variables starting from a (very) large set of independent variables.

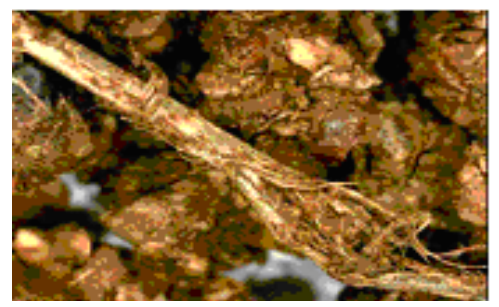

Olive husk sample before drying

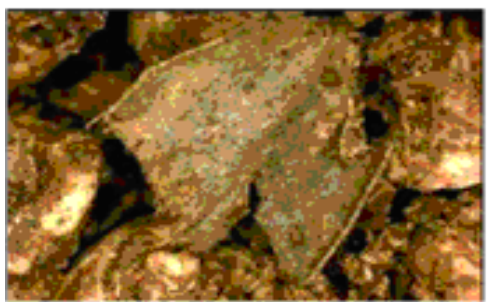

Olive husk sample after drying

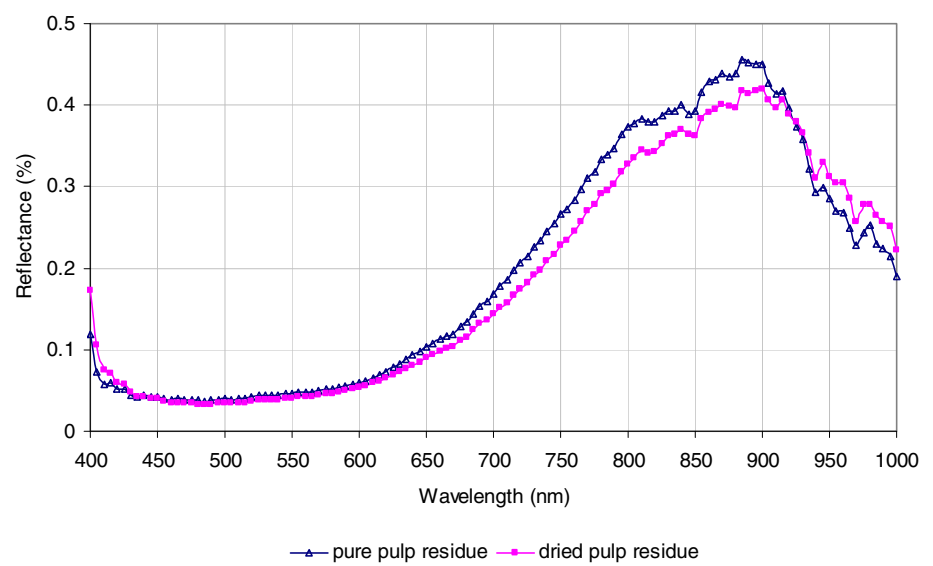

Fig. 4. Olive husk $(\mathrm{OH})$ samples average reflectance spectra in the VIS-NIR field (400-1000 nm), before and after drying, as detected by the HSI architecture

PLS regression is related to both Principal Component Regression (PCR) and MLR. PCR finds factors that capture the greatest amount of variance in the predictor $(X)$ variables (e.g. OH detected spectra). MLR seeks to find a single factor that best correlates predictor $(X)$ variables with predicted $(Y)$ variables. PLS attempts to find factors which both capture variance and achieve correlation. 
There are several ways to calculate PLS model parameters. Perhaps the most intuitive method is known as Non-Iterative PArtial Least Squares (NIPALS). NIPALS calculates scores $(T)$ and loadings $(P)$ (similar to those used in PCR), and an additional set of vectors known as weights $(W)$ (with the same dimensionality as the loadings $P$ ). Using NIPALS, the scores, weights, loadings and inner-coefficients are calculated sequentially. Before comparing the predictive ability of the models, it is useful to review several quality measures. In all of the measures considered, it was attempted to estimate the average deviation of the model from the data. The RootMean-Square Error of Calibration (RMSEC) tells us about the fit of the model to the calibration data. RMSEC is a measure of how well the model fits the data. The RootMean-Square Error of Cross-Validation (RMSECV) is a measure of a model's ability to predict samples that were not used to build the model. The RMSECV is typically refer to cross-validation experiments, where the existing calibration data set is split into different training and test sets in an effort to assess how a model built using all of the calibration data would perform when applied to new data.

\section{Results}

The analysis of the spectra demonstrates the high selectivity of the proposed approach to assess the presence of moisture in $\mathrm{OH}$, to discriminate about $\mathrm{OH}$ product morphometrical characteristics and, finally, to detect the presence of the different constituting elements (olive stones, pulp, olive peel and leaves).

\subsection{Olive Husk Moisture Detection}

The spectral plot clearly shows as the intensity of the spectral response of $\mathrm{OH}$ samples is influenced by moisture (Figure 4). Dry samples are characterized, in fact, by higher reflectance in the NIR region between $940 \mathrm{~nm}$ and $1000 \mathrm{~nm}$. The opposite is detectable in the VIS region $(400-700 \mathrm{~nm})$ and in the NIR region between $700 \mathrm{~nm}$ and $940 \mathrm{~nm}$. A multiple single band intensity comparison, at specific wavelengths, can thus be applied to perform the "simple" recognition of $\mathrm{OH}$ samples according to their moisture content. The results obtained applying the PLS modelling are reported in Figure 6. In the figure are also reported the trend of the root mean square error related to the calibration phase (RMSEC) (model generation) and the trend of the root mean square error for the validation (RMSECV) (Figure 6.1) performed in the entire investigated VIS-NIR spectra range. From the analysis of the plot (Figure 6.1) it is thus possible to see that after the first five latent variables the RMSECV slightly increases. Such a behavior being related to the "over-fitting" phenomena. Such a phenomenon usually occurs in multiple linear regression (MLR) when the number of factors gets too large, for example greater than the number of observations. In modelling terms it means that the greater is the increase of the RMSECV, the higher is the possibility that the obtained model, even if well fitting the sample data, fails when new data have to be predicted. 


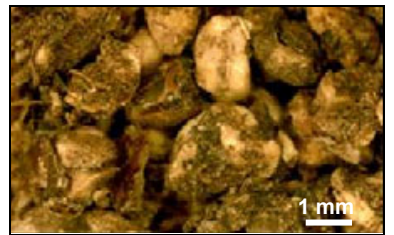

$-4.0+2.0 \mathrm{~mm}$

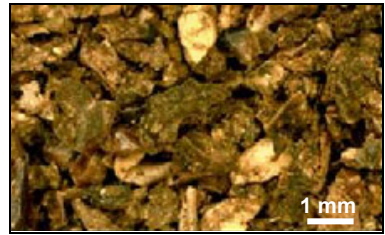

$-2.0+1.0 \mathrm{~mm}$

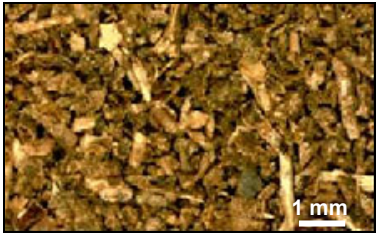

$-1.0+0.5 \mathrm{~mm}$

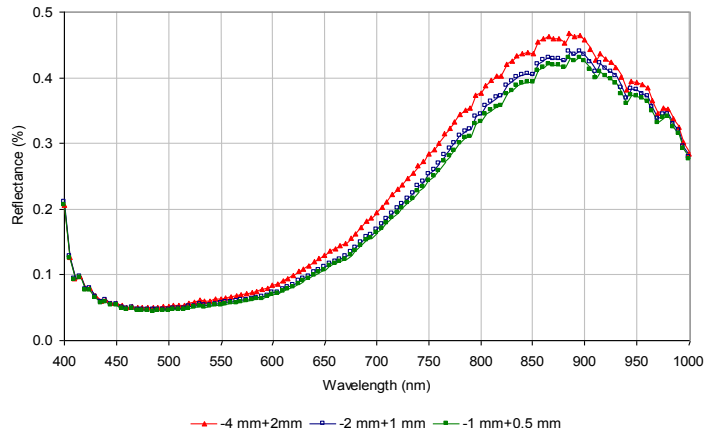

Fig. 5. Olive husk $(\mathrm{OH})$ size class samples average reflectance spectra in the VIS-NIR field (400-1000 nm), as detected by the HSI architecture

\subsection{Olive Husk Morphometrical Characteristics}

The results show as the HSI approach is able to perform a good discrimination of $\mathrm{OH}$ morphometrical characterization. The spectral plot (Figure 5) clearly outline as the spectra intensity decreases, decreasing the investigated $\mathrm{OH}$ size classes. Such a behaviour is detectable in the entire investigated wavelength range $(400-1000 \mathrm{~nm})$, being more marked in the NIR range between $800 \mathrm{~nm}$ and $950 \mathrm{~nm}$. As for the moisture a multiple single band intensity comparison, at specific wavelengths, can be carried out in order to perform a "simple" product morphological recognition. In Figure 7 the results obtained applying the PLS modelling are reported. Differently from the moisture case the RMSECV (Figure 7.1) practically remains constant for all the computed latent variables. Starting from $\mathrm{OH}$ collected spectra a good predictive model for $\mathrm{OH}$ morphometrical attributes can be thus built.

\subsection{Olive Husk Composition}

The HSI allows to perform a full characterization of $\mathrm{OH}$ in terms of constituents. The approach is able to well discriminate among olive stones, pulp, olive peel and leaves (Figure 8 ). The spectra are characterized by different intensity and signature, allowing this way to perform a correct identification of the different constituents.

Leaves. They are characterized, in spectral terms, by a signature that is completely different from olive stones, pulp and olive peel. The presence of a "valley" in the spectra in the VIS range $(760 \mathrm{~nm})$ allows to perform its detection/discrimination, from the other investigated constituents, just adopting a single band intensity comparison at specific wavelength (760 nm) (Figure 8). 


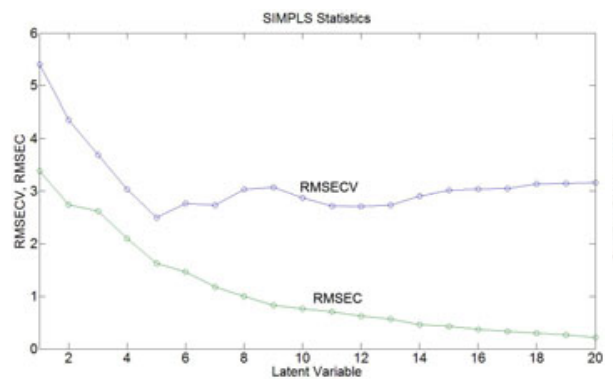

6.1

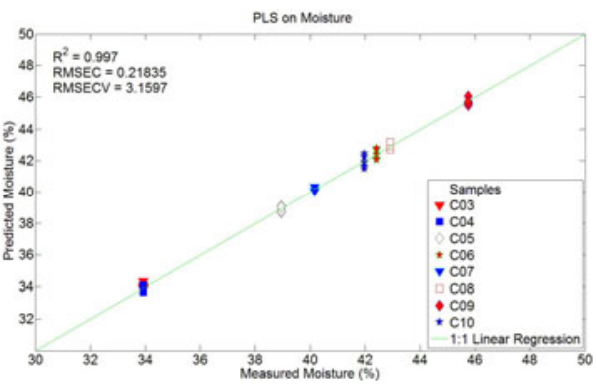

6.2

Fig. 6. Error plot (6.1) in function of the latent variables of the PLS modeling. RMSEC: root mean square error in calibration phase and RMSECV: root mean square error in validation. 6.2: prediction plot of both PLS model and its independent test as it results after olive husk $(\mathrm{OH})$ moisture content spectrophotometric data processing as resulting from the analyses performed in the VIS-NIR (400-1000 nm) wavelengths interval on several OH samples (Ci).

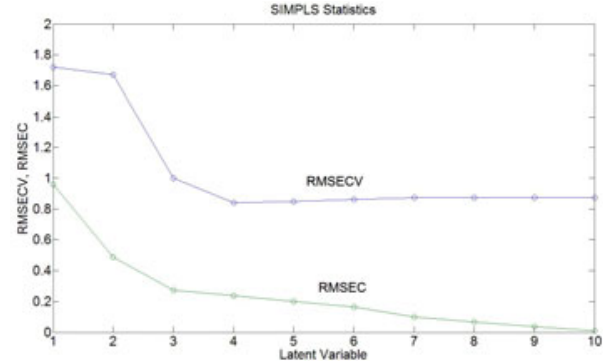

7.1

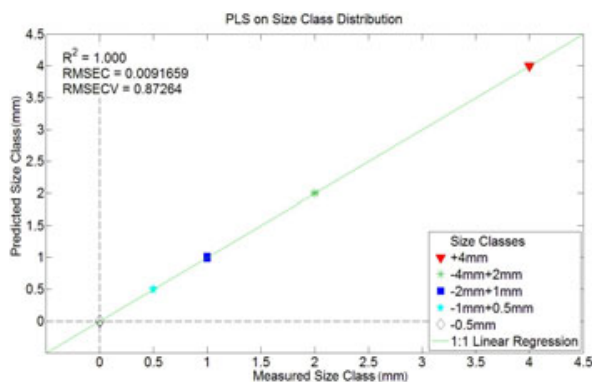

7.2

Fig. 7. Error plot (7.1) in function of the latent variables of the PLS modeling. RMSEC: root mean square error in calibration phase and RMSECV: root mean square error in validation. 7.2: prediction plot of both PLS model and its independent test as it results after olive husk $(\mathrm{OH})$ size class distribution spectrophotometric data processing as resulting from the analyses performed in the VIS-NIR (400-1000 nm) wavelengths interval.

Olive peel. This product is characterized by a spectral response similar to pulp but of a lower intensity. Furthermore in comparison with pulp, and their other constituents, it also presents a lower intensity increase of the spectra in the wavelength range between 550 and $650 \mathrm{~nm}$ (Figure 8). Both these aspects can be successfully applied to perform olive peel recognition adopting a multiple single band intensity comparison, at specific wavelengths, or a specific wavelengths intensity ratio analysis.

Pulp. Spectra are characterized by an average intensity value, in the wavelength range between $400 \mathrm{~nm}$ and $1000 \mathrm{~nm}$, lower than leaves and olive stones and higher than olive peel. Discrimination can be thus performed, in the previous mentioned interval, following single and/or multiple band intensity comparison at specific wavelengths. 
Olive stones. They are characterized by the higher spectral response in the VIS range between $550 \mathrm{~nm}$ and $700 \mathrm{~nm}$. In the NIR range their spectral response is quite similar to leaves. Between $830 \mathrm{~nm}$ and $840 \mathrm{~nm}$, differently from leaves presenting a progressive increase of the intensity of the spectral response, olive stone spectral intensity practically remains constant (Figure 8). Such a behaviour can be utilized in recognition adopting an intensity-based-gradient-discrimination approach, applied to the described interval, together with a single band intensity comparison, at specific wavelengths $(760 \mathrm{~nm})$.

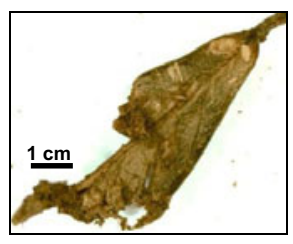

Leaf

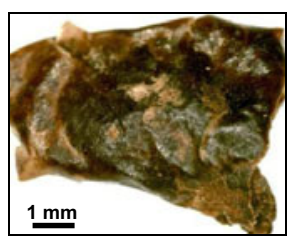

Olive peel

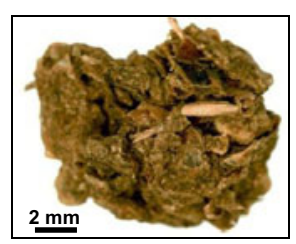

Olive pulp residue

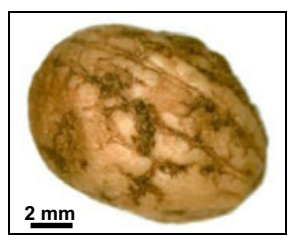

Olive stone

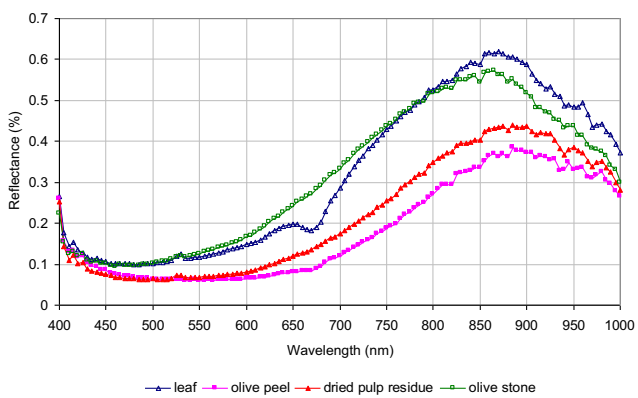

$\rightarrow$ leaf $\rightarrow$ olive peel $\rightarrow$-dried pulp residue $\rightarrow$ olive stone

Fig. 8. Spectral plots in the VIS-NIR field (400-1000 nm) of olive husk $(\mathrm{OH})$ product. Leaves, olive peel, dried pulp residue and olive stone are well characterized by their collected spectral firms.

\section{Conclusions}

The possibility to apply an HyperSpectral Imaging (HIS) based approach to perform a full characterization of olive husk $(\mathrm{OH})$ products addressed to maximize their optimal utilization as bio-masses was experimentally tested. Results showed as the HSI can be considered as an innovative, flexible and low cost tool that, combining imaging and reflectance spectroscopy, can be profitably utilized. More in details, the results obtained on different samples of olive husks $(\mathrm{OH})$, characterized by a different moisture content and different size class composition, allow to well discriminate these attributes. Furthermore the study demonstrated as the procedure is particularly suitable to be utilized to recognize the different $\mathrm{OH}$ constituting materials. Such a result is particularly important for a wider utilization of the proposed technique, and the related recognition logics, in other industrial sectors (production of polyols, furfural, olive seed oil, phenol-formaldehyde resins), and/or other applications (utilization of olive-mill waste on degraded agricultural soils, as bio-sorbers activated 
carbon from olive stone, as a plastic filler, as an abrasive, as a metal bio-sorbent, as dietary animal supplementation, etc.) in order to perform a full recovery of olive-mill waste, following different strategies finalized to obtain different marketable products.

\section{References}

[1] Matosa, M., Barreiro, M.F., Gandinia, A.: Olive Stone as a Renewable Source of Biopolyols. Industrial Crops and Products 32, 7-12 (2010)

[2] Niaounakis, M., Halvadakis, C.P.: Olive Processing Waste Management: Literature Review and Patent Survey. Elsevier, Amsterdam (2006)

[3] FAOSTAT, Website of Food and Agriculture organization of the United Nations (2009), http: / / faostat.fao.org/

[4] IOC, Web site of International Olive Council (2009), http: / /www. internationaloliveoil.org/

[5] Vlyssides, A.G., Loizides, M., Karlis, P.K.: Integrated Strategic Approach for Reusing Olive Oil Extraction by-Products. J. Cleaner Prod. 12, 603-611 (2004)

[6] Vlyssides, A.G., Barampouti, E.M.P., Mai, S.T.: Physical Characteristics of Olive Stone Wooden Residues: Possible Bulking Material for Composting Process. Biodegradation 19, 209-214 (2008)

[7] Durán, C.Y.: Propriedades Termoquímicas del Arujo de Aceitona. Poder calorífico. Grasas y Aceites 36, 45-47 (1985)

[8] Caballero, J.A., Conesa, J.A., Font, R., Marcilla, A.: Pyrolysis Kinetics of Almond Shells and Olive Stones Considering Their Organic Fractions. J. Anal. Appl. Pyrol. 42, 159-175 (1997)

[9] López, M.C.B., Blanco, C.G., Martínez-Alonso, A., Tascón, J.M.D.: Composition of Gases Released During Olive Stones Pyrolysis. J. Anal. Appl. Pyrol. 65, 313-322 (2002)

[10] Rios, R.V.R.A., Martínez-Escandel, M., Molina-Sabio, M., Rodríguez-Reinoso, F.: Carbon Foam Prepared by Pyrolysis of Olive Stones Under Steam. Carbon 44, 1448 1454 (2006)

[11] Skoulou, V., Swiderski, A., Yang, W., Zabaniotou, A.: Process characteristics and products of olive kernel high temperature steam gasification (HTSG). Bioresour. Technol. 100, 2444-2451 (2009)

[12] Goetz, A.F.H., Vane, G., Solomon, T.E., Rock, B.N.: Imaging Spectrometry for Earth Remote Sensing. Science 228, 1147-1153 (1985)

[13] Hege, E., O’Connell, D., Johnson, W., Basty, S., Dereniak, E.: Hyperspectral Imaging for Astronomy and Space Surveillance. In: Proceedings of the SPIE, vol. 5159, pp. 380-391 (2003)

[14] Wood, K.S., Gulian, A.M., Fritz, G.G., Van Vechten, D.: A QVD Detector for Focal Plane Hyperspectral Imaging. Astronomy. Bulletin of the American Astronomical Society 34, 1241 (2002)

[15] Monteiro, S., Minekawa, Y., Kosugi, Y., Akazawa, T., Oda, K.: Prediction of Sweetness and Amino Acid Content in Soybean Crops from Hyperspectral Imagery. ISPRS Journal of Photogrammetry and Remote Sensing 62(1), 2-12 (2007)

[16] Smail, V., Fritz, A., Wetzel, D.: Chemical Imaging of Intact Seeds with NIR Focal Plane Array Assists Plant Breeding. Vibrational Spectroscopy 42(2), 215-221 (2006)

[17] Uno, Y., Prasher, S., Lacroix, R., Goel, P., Karimi, Y., Viau, A.: Artificial Neural Networks to Predict Corn Yield from Compact Airborne Spectrographic Imager Data. Computers and Electronics in Agriculture 47(2), 149-161 (2005) 
[18] Lyon, R.C., Lester, D.S., Lewis, E.N., Lee, E., Yu, L.X., Jefferson, E.H.: Near-infrared Spectral Imaging for Quality Assurance of Pharmaceutical Products: Analysis of Tablets to Assess Powder Blend Homogeneity. AAPS PharmSciTech. 3(3), 17 (2002)

[19] Rodionova, O., Houmøller, L., Pomerantsev, A., Geladi, P., Burger, J., Dorofeyev, V.: NIR Spectrometry for Counterfeit Drug Detection: a Feasibility Study. Analytica Chimica Acta 549(1-2), 151-158 (2005)

[20] Roggo, Y., Edmond, A., Chalus, P., Ulmschneider, M.: Infrared Hyperspectral Imaging for Qualitative Analysis of Pharmaceutical Solid Forms. Analytica Chimica Acta 535(12), 79-87 (2005)

[21] Ferris, D., Lawhead, R., Dickman, E., Holtzapple, N., Miller, J., Grogan, S.: Multimodal Hyperspectral Imaging for the Non Invasive Diagnosis of Cervical Neoplasia. Journal of Lower Genital Tract Disease 5(2), 65-72 (2001)

[22] Kellicut, D., Weiswasser, J., Arora, S., Freeman, J., Lew, R., Shuman, C.: Emerging Technology: Hyperspectral Imaging. Perspectives in Vascular Surgery and Endovascular Therapy 16(1), 53-57 (2004)

[23] Zheng, G., Chen, Y., Intes, X., Chance, B., Glickson, J.D.: Contrast-enhanced Nearinfrared (NIR) Optical Imaging for Subsurface Cancer Detection. Journal of Porphyrins and Phthalocyanines 8(9), 1106-1117 (2004)

[24] Lu, R.F., Chen, Y.R.: Hyperspectral Imaging for Safety Inspection of Food and Agricultural Products. In: SPIE Conference on Pathogen Detection and Remediation for Safe Eating, Boston, USA (1998)

[25] Wold, S., Esbensen, K., Geladi, P.: Principal Components Analysis. Chemo. And Intell. Lab. Sys. 2, 37-52 (1987)

[26] Geladi, P., Kowalski, B.R.: PLS Tutorial. Anal. Chim. Acta. 185(1) (1986)

[27] Lorber, A.L., Wangen, E., Kowalski, B.R.: A Theoretical Foundation for the PLS Algorithm. J. Chemometrics 1(19) (1987)

[28] Martens, H., Næs, T.: Multivariate Calibration. John Wiley \& Sons, New York (1989)

[29] Höskuldsson, A.: Prediction Methods in Science and Technology. Thor Publishing, Denmark (1996) 